\title{
The Evaluation of Complexity Constants from Polarographic Data
}

\author{
ANDERS RINGBOM and LARS ERIKSSON \\ Institute of Analytical and Inorganic Chemistry, Abo Akademi, Abo, Finlaind
}

\begin{abstract}
The possibility of studying the equilibria of complex compounds polarographically was recognized by Heyrovsky and Ilkovic ${ }^{1}$ as early as 1935 . Since then several polarographic determinations of equilibrium constants of various systems have been reported in the literature.

Hitherto essentially the last complexity product of a system has been measured, whereas little attention has been paid to the consecutive constants illustrating the stepwise formation of complex compounds. Some authors even seem to deny the possibility of measuring these constants polarographically.

In 1950 the present authors ${ }^{2}$ suggested the use of the polarographic method for the investigation of the stepwise formation of complexes, and recently de Ford and Hume ${ }^{3}$ have presented a method of calculating the consecutive constants from polarographic data. Furthermore Hume, de Ford and Cave have reported their results on the equilibrium constants of the cadmium thiocyanate system.

De Ford and Hume ${ }^{3}$ assume that the ligand concentration at the drop surface is the same as the concentration in the body of the solution, and their calculations refer to the case where the central metal ion is reversibly reducible at the electrode. In this article we intend to show that the polarographic method is capable of still wider application. It can be used when the ligand concentrations at the drop surface and in the body of the solution are not the same and even when the central metal ion is not reversibly reducible. In the last case, however, it is necessary to use an indirect method based on the behaviour of an ,indicator ion".
\end{abstract}

\section{DIRECT POLAROGRAPHIC METHOD}

The method generally used for the determination of the last complexity product is based on the following principle:

Let $M$ be the central atom and $A$ the ligand of a complex compound $\mathbf{M A}_{\mathbf{n}}$. If $E_{1}$ is the half-wave potential of a solution containing no ligand - e.g. Acta Chem. Scand. 7 (1953) No. 7 
a metal perchlorate solution - and $E_{2}$ is the half-wave potential in the presence of a large excess of ligand, the following equation is valid:

$$
\Delta E_{\mathbf{z}}=E_{2}-E_{1}=-\frac{R T}{m F} \cdot \ln \left(\beta_{\mathfrak{n}} \cdot A^{\mathfrak{n}}\right)
$$

It is assumed that the $m$-valent metal is reduced to the metallic state. $\beta_{n}$ is the last complexity product of the following system of equilibria:

$$
\frac{\mathrm{MA}}{\mathrm{M} \cdot \mathrm{A}}=\beta_{1} ; \frac{\mathrm{MA}_{2}}{\mathrm{M} \cdot \mathrm{A}^{2}}=\beta_{2} ; \cdots \cdots ; \frac{\mathrm{MA}_{\mathrm{n}}}{\mathrm{M} \cdot \mathrm{A}^{\mathbf{n}}}=\beta_{\mathrm{n}}
$$

The letters denote concentrations of different species. For the sake of convenience, activity coefficients have not been taken into consideration. The relationship between the complexity products and the individual complexity constants is

where

$$
b_{1} \cdot b_{2} \cdots b_{n}=\beta_{n}
$$

$$
b_{n}=\frac{M_{n}}{M A_{n-1} \cdot A}
$$

Equation ( 1 ) is based on the assumption that the concentration of the ligand at the electrode is the same as in the bulk of the solution and that only the last of the consecutive complex compounds needs to be taken in account. In other words, the equation cannot be used unless the final complex is strong and there is a large excess of ligand in the solution.

If a more generally valid equation is required all the complexity equilibria must be taken in consideration, and it is further necessary to pay attention to the fact that the concentrations at the electrode surface and in the solution are not the same. Polarographic equilibrium calculations must always be made on the basis of the concentrations at the surface. According to general principles of polarography we can write:

$\begin{array}{lcc}\text { Total concentration of ligand } & \begin{array}{c}\text { Solution } \\ \mathbf{c}_{\mathrm{A}}\end{array} & \text { Electrode surface } \\ \text { Total concentration of metal } & \mathbf{c}_{\mathrm{M}} & \frac{i_{\mathrm{d}}-i}{i_{\mathrm{d}}} \cdot \mathbf{c}_{\mathrm{M}} \\ \text { Concentration of free ligand } & \mathbf{A} & \mathbf{A}_{\mathrm{o}} \\ \text { Concentration of free metal ion } & \mathbf{M} & \mathbf{M}_{\mathrm{o}}\end{array}$

The concentrations at the electrode surface are designated by the subscript $0, i$ is the measured current, $i_{\mathrm{d}}$ is the diffusion current. The total concentration of the ligand at the electrode surface is known, and the total concentration of the metal can be calculated if $i$ and $i_{\mathrm{d}}$ are also known. $\mathrm{A}_{\mathrm{o}}$ will be discussed later. $M_{0}$ can be calculated from the equation

$$
\Delta E=-\frac{R T}{m F} \cdot \ln \frac{\frac{i_{\mathrm{d}}-i}{i_{\mathrm{d}}} \cdot \mathrm{c}_{\mathrm{M}}}{\mathrm{M}_{\mathrm{o}}}
$$

Actta Chem. Scand. 7 (1953) No. 7 
$\Delta E$ is the difference between the potential of a metal perchlorate solution with ligand present and without any ligand at a defined current value. If $i=1 / 2 i_{\mathrm{d}}$, then $\Delta E=\Delta E_{1 / \mathrm{s}}$, the difference between half-wave potentials. Also other current values can be employed if the small corrections for the change in the residual current and the diffusion current (due to the changes in the diffusion coefficients) are taken into account.

If weak complexes are formed, the slope of the curve will usually be the theoretical one, but if the complex formed is strong and if only small amounts of the ligand are present, the slope, especially in the upper part of the curve, will be smaller. This is because $A_{o}$ increases when metal is removed from the solution. Consequently $\mathrm{M}_{\mathrm{o}}$ decreases as the current increases, and the curve shifts towards more negative values. Of course, the change in the slope is no indication of any irreversible reaction.

If consecutive complexity products are to be determined, $\Delta E$ must be measured at different ligand concentrations $\mathrm{c}_{\mathrm{A}}$. For the calculations, $\Delta E$ and $\mathbf{c}_{\mathrm{A}}$ must be expressed as functions of the equilibrium constants. This is easily done, since the total concentration equals the sum of the concentrations of all the components. In other words:

$$
\frac{i_{\mathrm{d}}-i}{i_{\mathrm{d}}} \cdot \mathrm{c}_{\mathrm{M}}=\mathrm{M}_{\mathrm{o}} \cdot\left(1+\beta_{1} \cdot \mathrm{A}_{\mathrm{o}}+\beta_{2} \cdot \mathrm{A}_{\mathrm{o}}^{2}+\cdots+\beta_{\mathrm{n}} \cdot \mathrm{A}_{\mathrm{o}}^{\mathrm{n}}\right)
$$

From (5) and (6) we obtain

$$
\Delta E=-\frac{R T}{m F} \cdot \ln \left(1+\beta_{1} \cdot \mathrm{A}_{\mathrm{o}}+\beta_{2} \cdot \mathrm{A}_{\mathrm{o}}^{2}+\cdots+\beta_{\mathfrak{n}} \cdot \mathrm{A}_{\mathrm{o}}^{\mathrm{n}}\right)
$$

Furthermore

$$
c_{\mathrm{A}}=\mathrm{A}_{\mathrm{o}}+\mathrm{M}_{\mathrm{o}} \cdot\left(\beta_{1} \cdot \mathrm{A}_{\mathrm{o}}+2 \cdot \beta_{2} \cdot \mathrm{A}_{\mathrm{o}}^{2}+\cdots+\mathrm{n} \cdot \beta_{\mathrm{n}} \cdot \mathrm{A}_{\mathrm{o}}^{\mathrm{n}}\right)
$$

The problem of computing individual complexity constants is hereby reduced to a purely mathematical problem. One simply conducts a series of $\mathrm{c}_{\mathrm{A}}$ and $\Delta E$ measurements and calculates $\mathrm{A}_{\mathrm{o}}$ and the $\beta$ constants from equations (5), (7) and (8). If $n=1$ or 2 , the calculations are very simple. If $n$ is larger than 2, equations of higher order are obtained, and their solution is not quite simple. An approximate value for $\beta_{1}$ can be obtained from measurements at a low ligand concentration by assuming that only the first complex is formed. In an analogous way an approximate value for the last complexity product $\beta_{n}$ can be obtained - if the complex is strong enough - from measurements at high ligand concentrations. Equation (7) will then be transformed into

$$
\Delta E=-\frac{R T}{m F} \cdot \ln \left(\beta_{\mathrm{n}} \cdot \mathrm{A}_{\mathrm{o}}^{\mathrm{n}}\right)
$$

This equation is identical with the previous equation (1), which neglects all the complexes with less than $n$ ligands.

Correct values for the other constants can be obtained by successive approximations. Leden ${ }^{5}$, in a potentiometric study of the cadmium complexes, 
was the first to determine consecutive complexity constents by measuring the concentration of the central metal ion. He used a partly graphical method. Fronaeus ${ }^{6}$ has later modified this method. De Ford and Hume ${ }^{3}$ used a method similar to Leden's. For details one must refer to the original papers and to a following paper by Eriksson?

De Ford and Hume assume that the ligand concentration at the drop surface is the same as in the body of the solution. It may be remarked that this assumption is not essential. According to equations (5)-(8) it is possible to determine all concentrations in question at the drop surface.

It may some time be of advantage to measure the potential shift at a smaller current than $1 / 2 i_{\mathrm{d}}$.

In the following paper ${ }^{7}$, the polarographic determinations of the complexity constants of cadmium chloride and bromide will be described.

If the central metal ion is not reversibly reducible or oxidizable but the ligand is, the consecutive complexity constants can be determined polarographically in an analogous way. The calculation will then be simpler and can be made according to the method of J. Bjerrum ${ }^{8}$.

\section{INDIRECT POLAROGRAPHIC METHOD}

Even if polarography offers a possibility of determining individual complexity constants, the method seems at first sight to be of only limited value as the application of polarographic methods to equilibrium problems presupposes a reversible reaction at the electrode surface. Unfortunately, only a few metals - e.g. $\mathrm{Cd}, \mathrm{Pb}, \mathrm{Tl}$ - are reversibly reducible at the mercury electrode.

It may be noted, however, that a non-reducible metal can be titrated amperometrically after adding a suitable indicator ion, as shown by Ringbom and Wilkman ${ }^{\theta}$, and in an analogous way it is possible to determine the concentration of a non-reducible metal ion by using another ion as an indicator.

As an example we may take the case in which the equilibrium constants of the compounds of a metal $M$ and the ligand $A$ are to be determined. If cadmium, for example, forms complexes of known strength with the same ligand, the cadmium ion can be used as an indicator, provided that the cadmium wave appears before the $M$-wave.

Applying the equation (7) to cadmium we get

$$
\Delta E=-\frac{R T}{2 F} \cdot \ln \left(1+\beta_{1} \cdot \mathrm{A}_{0}+\beta_{2} \cdot \mathrm{A}_{0}^{2}+\cdots+\beta_{\mathrm{n}} \cdot \mathrm{A}_{0}^{\mathrm{n}}\right)
$$

If all the $\beta$ constants are known, the equation (10) represents a quite general method for the determination of anion (or molecule) concentrations. One merely adds a little indicator, in this case cadmium perchlorate, to the metal solution and measures the half-wave potential with reference to the half-wave potential of a cadmium perchlorate polarogram. From a graph showing the relation between $\Delta E$ and $\mathrm{A}_{0}$ the ligand concentration can be immediately obtained. 


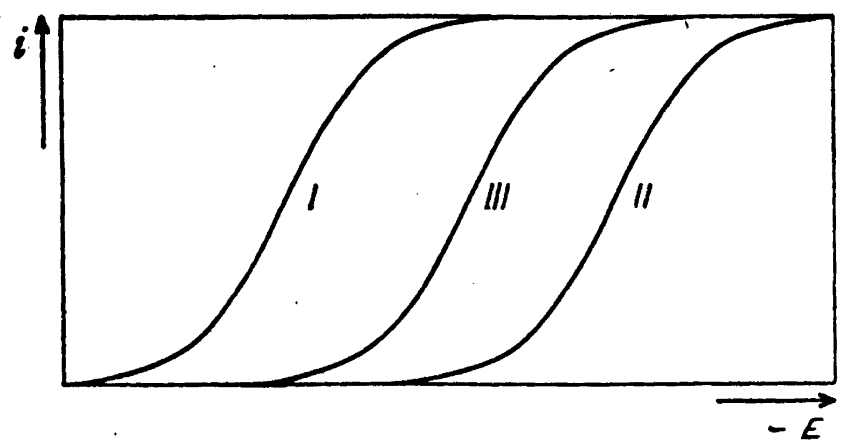

Fig. 1. Polarograms illustrating the indicator method of measuring ligand concentrationis.

I. Indicator ion.

II. Indicator ion + ligand.

III. Indicator ion + metal ion + ligand.

It is clear that when $\mathrm{M}$ ions are added to a solution containing $\mathrm{Cd}$ ions and A ligands, they will react with part of the ligands; in other words, $A_{0}$ will decrease. In Fig. 1 three polarograms are plotted: curve I has been recorded in a cadmium perchlorate solution, curve II has been taken in a solution containing cadmium and the ligand, and curve III in the same solution as II after adding $M$ ions in a known concentration.

It may be emphasized that the mathematical calculation of the complexity constants by the indirect method is simpler than in the direct determination of the central ion. It is suitable to use the average ligand number $\overline{\mathbf{n}}$ introduced by J. Bjerrum ${ }^{8}$ i.e.

$$
\overline{\mathbf{n}}=\frac{\text { number of bound ligands }}{\text { total number of metal atoms }}
$$

The average ligand number of cadmium at the electrode surface can be calculated from the equation

$$
\overline{\mathrm{n}}_{\mathrm{oCd}}=\frac{\beta_{1} \cdot \mathrm{A}_{\mathrm{o}}+2 \cdot \beta_{2} \cdot \mathrm{A}_{\mathrm{o}}^{2}+\cdots+\mathrm{n} \cdot \beta_{\mathrm{n}} \cdot \mathrm{A}_{\mathrm{o}}^{\mathrm{n}}}{1+\beta_{1} \cdot \mathrm{A}_{\mathrm{o}}+\beta_{2} \cdot \mathrm{A}_{\mathrm{o}}^{2}+\cdots+\beta_{\mathrm{n}} \cdot \mathrm{A}_{\mathrm{o}}^{\mathrm{n}}}
$$

It is convenient to plot $\bar{n}_{\mathrm{ocd}}$ as a function of $\log A_{0}$. If only mononuclear complexes are formed, $\bar{n}_{\mathrm{ocd}}$ does not depend on $c_{c d}$ and $\overline{\mathrm{n}}_{\mathrm{oM}}$ can then be computed from the equation

$$
\overline{\mathrm{n}}_{\mathrm{oM}}=\frac{\mathrm{c}_{\mathrm{A}}-\mathrm{A}_{\mathrm{o}}-\overline{\mathrm{n}}_{\mathrm{oCd}} \cdot \frac{i_{\mathrm{d}}-i}{i_{\mathrm{d}}} \cdot \mathrm{c}_{\mathrm{Cd}}}{\mathrm{c}_{\mathrm{M}}}
$$

For the complexity constants of the $M-A$-complexes the following method suggested by J. Bjerrum ${ }^{8}$ can be used:

Acta Chem. Scand. 7 (1953) No. 7 
Equation (12) is written as follows

$\overline{\mathrm{n}}_{\mathrm{OM}}+\left(\overline{\mathrm{n}}_{\mathrm{o}}-1\right) \cdot \mathrm{A}_{\mathrm{o}} \cdot \beta_{1}+\left(\overline{\mathrm{n}}_{\mathrm{o}}-2\right) \cdot \mathrm{A}_{\mathrm{o}}^{2} \cdot \beta_{2}+\cdots+\left(\overline{\mathrm{n}}_{\mathrm{o}}-\mathrm{n}\right) \cdot \mathrm{A}_{\mathrm{o}}^{\mathrm{n}} \cdot \beta_{\mathrm{n}}=0$

From the $\log A_{o}$ versus $\bar{n}_{O M}$ curve, $A_{o}$ values corresponding to $\bar{n}_{O M}=0.5$, 1.5, 2.5 etc. are taken. The constants are then calculated from these values by means of equation (14). Some approximations can usually be made.

When cadmium is used as an indicator, it seems probable that the concentrations of the following ligands can be determined: chloride, bromide, iodide, nitrite, thiocyanate, azide, acetate, several organic anions, ammonia. Cyanide ions form a strong complex with cadmium, but according to Kolthoff and Lingane ${ }^{10}$, the wave is not perfectly reversible. The complexes with nitrate, chlorate, bromate and fluoride seem to be too weak for the purpose in question.

- In the indirect method suggested above there is one weak point. If the metal $M$ is not reversibly reducible, the reaction between $M$ and the ligand does not occur instantaneously. Consequently, even if it is possible to calculate all the concentrations at the electrode surface, it is not certain that the $M$ ions are in equilibrium with the ligands liberated at the surface.

If $A$ and $A_{0}$ do not differ very much, i.e. if the slope of the curve is the theoretical one, the error caused by the unattained equilibrium can be neglected. All the cadmium complexes mentioned above; except the cyanide complex, seem to be so weak that no considerable differences between $A$ and $A_{0}$ exist if $i=1 / 2 i_{\mathrm{d}}$ or less. But if there is a marked decrease in the slope, then it is better to measure potential differences using a lower current strength than the half-wave current, or to measure $\Delta E$ at various current strengths and to extrapolate the values to $i=0$. In this way the concentration in the bulk of the solution is obtained, and the polarographic method is similar to a potentiometric method, the indicator system representing an electrode of the second order.

The accuracy of the method described above is of course dependent on the strength of the indicator complex. If the indicator complex is a very weak one, the accuracy is low, but if the complex is strong, the attainable accuracy can be surprisingly high. A simple calculation illustrates this.

In direct measurements of cadmium potentials, the error at $25^{\circ}$ is

$$
\frac{\mathrm{d} E}{\frac{\mathrm{dCd}}{\mathrm{Cd}}}=\frac{R T}{2 F}=12.3 \mathrm{mV}
$$

This means that $\pm 1 \mathrm{mV}$ corresponds to an error about $\pm 8 \%$. If on the other hand, the concentration of ligand $A$ is measured by means of cadmium potentials, the error is given by the equation

$$
\frac{\mathrm{d} E}{\frac{\mathrm{dA}}{\mathrm{A}}}=-\overline{\mathrm{n}}_{\mathrm{Cd}} \cdot \frac{R T}{2 F}
$$

Acta Chem. Scand. 7 (1953) No. 7 
This means that if the average ligand number $\bar{n}_{\mathrm{cd}}$ is larger than 1 , the theoretical accuracy is greater than in the direct measurement of cadmium concentrations. If cadmium forms a strong complex, so that $\overline{\mathbf{n}}_{\mathrm{Cd}}$ approaches the value 4 , then $\pm 1 \mathrm{mV}$ will correspond to an error of only $\pm 2 \%$. Of course, other factors can affect the results, which largely depend on the accuracy of the determination of the indicator complexity constants. It may also be mentioned that if the M-complexes are weak, the accuracy in determining $\overline{\mathbf{n}}_{\mathbf{M}}$ and hence the equilibrium constants according to equation (13) is essentially dependent on the accuracy of the determination of the difference $c_{A}-A_{o}$. Consequently, it is necessary to have a rather high concentration of $M$ in order to decrease $A_{0}$ sufficiently.

The influence of activity coefficients has not been considered above. If possible, it is advantageous to make all the measurements in a concentrated sodium perchlorate solution, as is usual in potentiometric measurements.

The polarographic method is probably in some instances superior to the potentiometric method, since the dropping mercury electrode is more convenient than amalgam electrodes, which demand great care. Moreover, when low concentrations are measured, the polarographic method gives no polarization troubles. Which of the two methods can be used depends on the case in question; this aspect will not be discussed here. It seems, however, worth while to emphasize that polarography as well as potentiometry offer possibilities for the measurement of the concentrations of anions or molecule groups that form complexes with a reversibly reducible metal. It also appears to be theoretically possible to determine consecutive complexity constants of other metal complex compounds, even when neither the central ion nor the ligand can be determined polarographically.

\section{SUMMARY}

The possibility of determining consecutive complexity constants from polarographic data are discussed.

An indicator method is proposed for the determination of ligand concentrations. Complexity constants can be determined polarographically by this method even if neither the metal nor the ligand of the complex is reduced at the electrode.

\section{REFERENCES}

1. Heyrovsky, J. and Ilkovic, D. Collection Czech. Chem. Communs 7 (1935) 198.

2. Ringbom, A. Sjunde Nordiska Kemistmötet, Helsingfors (1950), p. 176.

3. de Ford, D. D. and Hume, D. N. J. Am. Chem. Soc. 73 (1951) 5321.

4. Hume, D. N., de Ford, D. D., and Cave, G. C. B. J. Am. Chem. Soc. 73 (1951) 5323.

5. Leden, I. Thesis, Lund (1943).

6. Fronaeus, S. Thesis, Lund (1948).

7. Eriksson, L. Acta Chem. Scand. 7 (1953). In press.

8. Bjerrum, J., Thesis, Copenhagen (1941).

9. Ringbom, A. and Wilkman, B. Acta Chem. Scand. 3 (1949) 22.

10. Kolthoff, I. M. and Lingane, J. J. Polarography, 2nd ed. New York (1952) 510.

Received June 19, 1953.

Acta Chem. Scand. 7 (1953) No. 7 